EPJ Web of Conferences 106, 03009 (2016)

DOI: $10.1051 /$ epjconf/201610603009

(C) Owned by the authors, published by EDP Sciences, 2016

\title{
Evaluation of Geometric Progression (GP) Buildup Factors using MCNP Codes (MCNP6.1 and MCNP5-1.60)
}

\author{
Kyung-O Kimª, Gyuhong Roh, and Byungchul Lee \\ Korea Atomic Energy Research Institute, 1045 Daedeok-daero, Yuseong-gu, Daejeon, Korea
}

\begin{abstract}
The gamma-ray buildup factors of three-dimensional point kernel code (QADCGGP) are re-evaluated by using MCNP codes (MCNP6.1 and MCNPX5-1.60) and ENDF/B-VI.8 photoatomic data, which cover an energy range of $0.015-15 \mathrm{MeV}$ and an iron thickness of 0.5-40 Mean Free Path (MFP). These new data are fitted to the Geometric Progression (GP) fitting function and are then compared with ANS standard data equipped with QAD-CGGP. In addition, a simple benchmark calculation was performed to compare the QAD-CGGP results applied with new and existing buildup factors based on the MCNP codes. In the case of the buildup factors of low-energy gamma-rays, new data are evaluated to be about 5\% higher than the existing data. In other cases, these new data present a similar trend based on the specific penetration depth, while existing data continuously increase beyond that depth. In a simple benchmark, the calculations using the existing data were slightly underestimated compared to the reference data at a deep penetration depth. On the other hand, the calculations with new data were stabilized with an increasing penetration depth, despite a slight overestimation at a shallow penetration depth.
\end{abstract}

\section{Introduction}

Codes based on the point kernel method have been widely used to analyze the radiation shielding and gamma-ray fields around radioactive sources. Particularly, QAD-CGGP [1] is a three-dimensional point kernel code employed using a double precision Combinatorial Geometry (CG) scheme and a more accurate Geometric Progression (GP) fitting function for the ANS-6.4.3 gamma-ray buildup factor [2]. However, ANS standard data only include single-material buildup factors that were last evaluated about 20 years ago. Hence, it is necessary to update existing data and supplement buildup factors for compound materials now widely used in radiation shielding.

In this study, the most widely used Monte Carlo codes (MCNP6.1 [3] and MCNP5-1.60 [4]) are used to derive gamma-ray buildup factors covering an energy range of $0.015-15 \mathrm{MeV}$ and an iron thickness of 0.5-40 Mean Free Path (MFP). These new data are compared with ANS standard data equipped with QAD-CGGP. In addition, a simple benchmark calculation was performed to compare the QAD-CGGP results applied with new and existing buildup factors, on the basis of MCNP6.1.

\footnotetext{
${ }^{\mathrm{a}}$ Corresponding author: k5kim@kaeri.re.kr
}

This is an Open Access article distributed under the terms of the Creative Commons Attribution License 2.0, which permits unrestricted use, distribution, and reproduction in any medium, provided the original work is properly cited. 


\section{Evaluation of Gamma-ray Buildup Factor}

The gamma-ray interaction with round mediums can be classified as the Compton scattering, Photoelectric effect, Pair production, Rayleigh scattering, and Photonuclear interaction [5], which have to be considered in radiation shielding and radiological effect. Because the relative importance of these interactions depends on incidental gamma-ray energy and the atomic number $\mathrm{Z}$ of the material, accurate calculation considering all interactions is a difficult and complex problem. However, it can be simply solved by introducing the buildup factor, which considers the influence of any scattered and secondary gamma-rays in the medium. For this reason, the buildup factor is widely used by point kernel shielding codes to account for scattered and secondary radiation in the medium.

The data for buildup factors are generally fitted to various curves (e.g., Taylor and polynomial functions) to estimate the values between a series of data points. The GP fitting function was first introduced to QAD-CGGP to provide a better fit for gamma-ray buildup factors and this function is defined as follows:

$$
\begin{array}{cc}
B(E, x)=1+(b-1)\left(K^{x}-1\right) /(K-1) & \text { for } K \neq 1 \\
=1+(b-1) x & \text { for } K=1
\end{array}
$$

where $E$ is the gamma-ray source energy, $x$ is the source-detector distance in the unit of MFP, $b$ is the buildup factor at $1 \mathrm{MFP}$, and $K(E, x)$ is the geometric ratio. The variation of parameter $K(E, x)$ according to the penetration depth represents the dose rate multiplication and change in the shape of the gamma-ray spectrum at $1 \mathrm{MFP}$. These GP parameters from 0.5 to $40 \mathrm{MFP}$ were previously established from ANS-6.4.3 gamma-ray buildup factors, and the rest of the $K(E, x)$ values $(<60 \mathrm{MFP})$ are extrapolated from those data using this formula;

$$
\begin{aligned}
K(x)= & 1+\left[K\left(x_{i}\right)-1\right] \times \exp \left\{\left[1-\left(x / x_{i}\right)^{1 / n}\right] /\left[1-\left(x_{j} / x_{i}\right)^{1 / n}\right]\right. \\
& \left.\times \ln \left[\left(K\left(x_{j}\right)-1\right) /\left(K\left(x_{i}\right)-1\right)\right]\right\}
\end{aligned}
$$

where $x_{i}=35 \mathrm{MFP}, x_{j}=40 \mathrm{MFP}$, and $n=10$. This study derives gamma-ray buildup factors of single iron material that covers an energy range of $0.015-15 \mathrm{MeV}$ and penetration depth of $0.5-40 \mathrm{MFP}$. The most widely used Monte Carlo codes (MCNP6.1 and MCNP5-1.60) and ENDF/B-VI.8 photoatomic data are used for this calculation, and the total linear attenuation factor is first analyzed to investigate the gamma-ray MFP (thickness) in the iron medium (see Fig. 1). As shown in the figure, this factor is exponentially decreased with increasing gamma-ray source energy and there is no significant difference between ENDF/B-VI.8 and ANS 6.4.3 cross-section data.

By using the total linear attenuation factor derived from this study, the buildup factors are obtained as a ratio of the total gamma-ray flux to the un-collided gamma-ray flux at each MFP and are fitted to the above-mentioned functions to analyze the GP parameters. $2 \times 10^{7}$ source particles are transported in these calculations using two versions of MCNP code, and the relative error of calculation results is less than 5\%. Table 1 represents the comparison of existing and new GP parameters. Figure 2 shows the buildup factors for low $(0.015 \mathrm{MeV})$, medium $(1 \mathrm{MeV})$, and high $(10 \mathrm{MeV})$ energy gamma-ray source, respectively. In the case of a low-energy gamma-ray, new data appear to be about $5 \%$ higher than the existing parameter. In other cases, two new data sets present a similar trend based on the specific penetration depth, while existing data continuously increase beyond that depth. In addition, it is expected that the buildup factors obtained from two Monte Carlo codes are slightly different from each other in large MFP because MCNP6.1 code is appropriately corrected for Compton Doppler broadening [6]. 


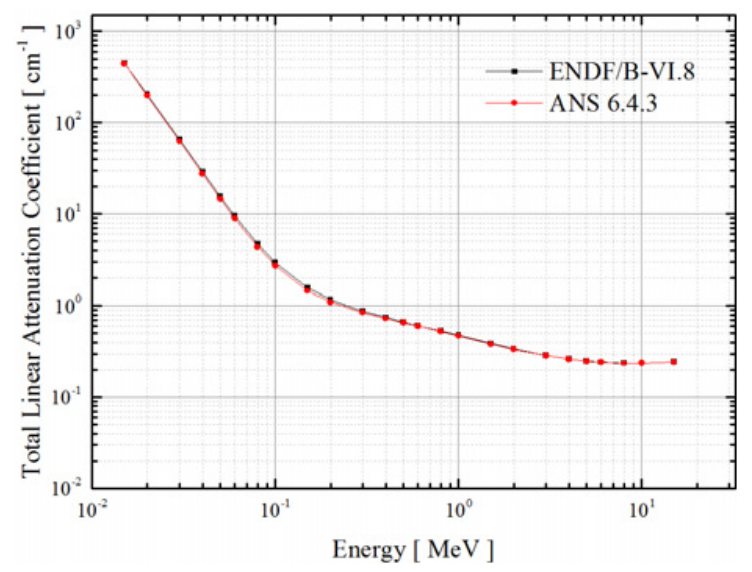

Figure 1. Total Linear Attenuation Coefficient derived from ENDF/B-VI.8 and ANS 6.4.3 Cross-section Data.

Table 1. Comparison of Existing and New GP Parameters.

\begin{tabular}{ccccccc}
\hline & Energy $[\mathrm{MeV}]$ & $b$ & $c$ & $a$ & $X_{k}$ & $d$ \\
\hline & 0.015 & 1.004 & 1.561 & -0.554 & 5.60 & 0.352 \\
\cline { 2 - 6 } & 0.05 & 1.099 & 0.366 & 0.232 & 14.01 & -0.135 \\
\cline { 2 - 6 } Existing Data & 0.10 & 1.389 & 0.557 & 0.144 & 14.11 & -0.079 \\
\cline { 2 - 6 } (ANS 6.4.3) & 0.50 & 1.957 & 1.261 & -0.046 & 24.77 & 0.008 \\
\cline { 2 - 6 } & 1.00 & 1.841 & 1.250 & -0.048 & 19.49 & 0.014 \\
\cline { 2 - 6 } & 5.00 & 1.483 & 1.009 & 0.012 & 13.12 & -0.026 \\
\cline { 2 - 6 } & 10.00 & -0.292 & 1.297 & 0.949 & 0.042 & 13.97 \\
\cline { 2 - 6 } & 0.015 & 1.024 & 0.333 & 0.069 & 16.72 & 0.245 \\
\cline { 2 - 6 } & 0.05 & 1.076 & 0.356 & 0.218 & 25.21 & -0.485 \\
New Data & 0.10 & 1.354 & 0.530 & 0.146 & 23.05 & -0.200 \\
\cline { 2 - 6 } (ENDF/B-VI.8 & 0.50 & 2.040 & 1.264 & -0.048 & 31.02 & -0.006 \\
\cline { 2 - 6 } and MCNP6) & 1.00 & 1.910 & 1.361 & -0.083 & 11.15 & 0.049 \\
\cline { 2 - 6 } & 5.00 & 1.613 & 0.966 & 0.023 & 19.53 & -0.053 \\
\hline New Data & 10.00 & 1.522 & 0.924 & 0.046 & 62.75 & -0.988 \\
\hline & 0.015 & 1.024 & 0.347 & 0.059 & 14.93 & 0.206 \\
\hline & 0.05 & 1.076 & 0.359 & 0.210 & 28.23 & -0.502 \\
\hline (ENDF/B-VI.8 & 0.10 & 1.353 & 0.516 & 0.160 & 39.38 & -0.900 \\
\cline { 2 - 6 } and MCNP5) & 0.50 & 2.040 & 1.231 & -0.039 & 30.87 & -0.040 \\
\cline { 2 - 6 } & 1.00 & 1.910 & 1.391 & -0.093 & 10.62 & 0.063 \\
\cline { 2 - 6 } & 5.00 & 1.613 & 0.963 & 0.023 & 35.63 & -0.195 \\
\hline & 10.00 & 1.521 & 0.897 & 0.055 & 33.95 & -0.309 \\
\hline
\end{tabular}

\section{Benchmark Calculation}

The simple benchmark simulation shown in Fig. 3 is performed to compare the accuracy of these buildup factors on the basis of the reference calculation (MCNP6.1). The problem is simply composed of the $5 \mathrm{~cm}$ radius concrete sphere surrounded by a single iron medium, and cobalt-60 $\left({ }^{60} \mathrm{Co}\right)$ isotopes are mixed with the concrete sphere positioned in the center of calculation geometry. The source strength is normalized to $1 \mathrm{Ci}\left(\mathrm{ASO}=3.7 \times 10^{10}\right.$ decays per second), and energies of two gamma-rays emitted from ${ }^{60} \mathrm{Co}$ isotope are $1.1732 \mathrm{MeV}$ and $1.3324 \mathrm{MeV}$, respectively. The volume of the spherical source is also divided into the 10 meshes along the radius $(r)$ and polar $(\theta) \&$ azimuthal $(\varphi)$ angles.

Table 2 shows the calculation results for the simple benchmark simulation shown in Fig. 3 . The three calculation codes are used for this benchmark, and the QAD-CGGP calculation is applied with existing and new buildup factors. The QAD-CGGP calculation employed using the existing data are slightly underestimated compared with the reference data at a deep penetration depth. On the other 


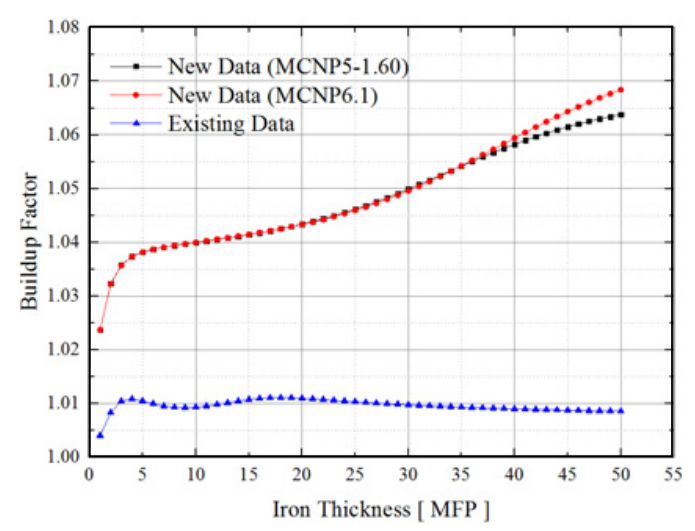

(a) $0.015 \mathrm{MeV}$

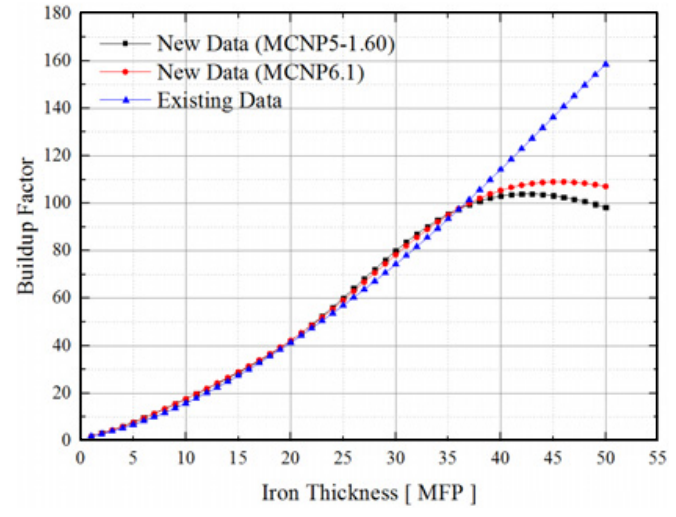

(b) $1.0 \mathrm{MeV}$

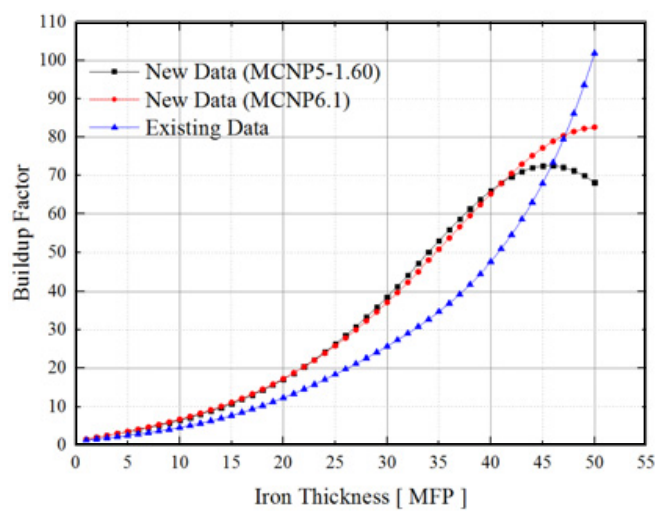

(c) $10.0 \mathrm{MeV}$

Figure 2. Buildup Factors as a Function of Gamma-ray Source Energy (0.015 MeV, $1.0 \mathrm{MeV}$, and $10.0 \mathrm{MeV})$.

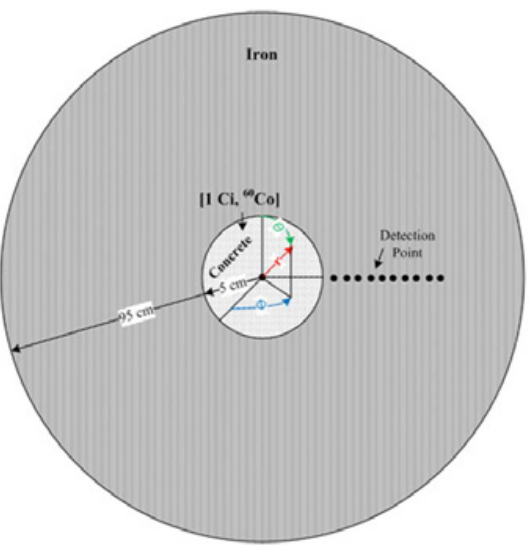

Figure 3. Calculation Geometry for Simple Benchmark Problem. 
Table 2. Dose Rate Distribution as a Function of Penetration Depth [Unit: $\mu \mathrm{Sv} / \mathrm{hr}]$.

\begin{tabular}{|c|c|c|c|c|}
\hline \multirow{2}{*}{$\begin{array}{l}\text { Iron Thickness } \\
{[\mathrm{cm}]}\end{array}$} & \multicolumn{3}{|c|}{ QAD-CGGP } & \multirow{2}{*}{$\begin{array}{l}\text { MCNP6.1* } \\
\text { (Rel. Error) }\end{array}$} \\
\hline & $\begin{array}{l}\text { Existing Data } \\
\text { (Value/Ref.) }\end{array}$ & $\begin{array}{l}\text { MCNP5 Data } \\
\text { (Value/Ref.) }\end{array}$ & $\begin{array}{l}\text { MCNP6 Data } \\
\text { (Value/Ref.) }\end{array}$ & \\
\hline 10 & $\begin{array}{c}2.72 \mathrm{E}+04 \\
(1.05)\end{array}$ & $\begin{array}{c}3.05 \mathrm{E}+04 \\
(1.17)\end{array}$ & $\begin{array}{c}3.09 \mathrm{E}+04 \\
(1.19)\end{array}$ & $\begin{array}{c}2.60 \mathrm{E}+04 \\
(3.46 \%)\end{array}$ \\
\hline 20 & $\begin{array}{c}2.87 \mathrm{E}+02 \\
(0.98) \\
\end{array}$ & $\begin{array}{c}3.37 \mathrm{E}+02 \\
(1.15)\end{array}$ & $\begin{array}{c}3.25 \mathrm{E}+02 \\
(1.11) \\
\end{array}$ & $\begin{array}{c}2.92 \mathrm{E}+02 \\
(2.97 \%) \\
\end{array}$ \\
\hline 30 & $\begin{array}{c}3.60 \mathrm{E}+00 \\
(0.98)\end{array}$ & $\begin{array}{c}4.41 \mathrm{E}+00 \\
(1.21)\end{array}$ & $\begin{array}{c}4.03 \mathrm{E}+00 \\
(1.10)\end{array}$ & $\begin{array}{c}3.66 \mathrm{E}+00 \\
(1.89 \%)\end{array}$ \\
\hline 40 & $\begin{array}{c}4.87 \mathrm{E}-02 \\
(0.96)\end{array}$ & $\begin{array}{c}6.16 \mathrm{E}-02 \\
(1.21)\end{array}$ & $\begin{array}{c}5.59 \mathrm{E}-02 \\
(1.10)\end{array}$ & $\begin{array}{l}5.09 \mathrm{E}-02 \\
(2.47 \%)\end{array}$ \\
\hline 50 & $\begin{array}{c}6.91 \mathrm{E}-04 \\
(0.99)\end{array}$ & $\begin{array}{c}8.78 \mathrm{E}-04 \\
(1.26)\end{array}$ & $\begin{array}{c}8.28 \mathrm{E}-04 \\
(1.19)\end{array}$ & $\begin{array}{l}6.95 \mathrm{E}-04 \\
(3.27 \%)\end{array}$ \\
\hline 60 & $\begin{array}{c}1.01 \mathrm{E}-05 \\
(0.93)\end{array}$ & $\begin{array}{c}1.27 \mathrm{E}-05 \\
(1.16)\end{array}$ & $\begin{array}{c}1.24 \mathrm{E}-05 \\
(1.13)\end{array}$ & $\begin{array}{l}1.09 \mathrm{E}-05 \\
(5.08 \%)\end{array}$ \\
\hline 70 & $\begin{array}{c}1.53 \mathrm{E}-07 \\
(0.91)\end{array}$ & $\begin{array}{c}1.87 \mathrm{E}-07 \\
(1.11)\end{array}$ & $\begin{array}{c}1.82 \mathrm{E}-07 \\
(1.08)\end{array}$ & $\begin{array}{c}1.68 \mathrm{E}-07 \\
(7.20 \%)\end{array}$ \\
\hline 80 & $\begin{array}{c}2.36 \mathrm{E}-09 \\
(0.89)\end{array}$ & $\begin{array}{c}2.77 \mathrm{E}-09 \\
(1.05)\end{array}$ & $\begin{array}{c}2.62 \mathrm{E}-09 \\
(0.99)\end{array}$ & $\begin{array}{c}2.64 \mathrm{E}-09 \\
(8.89 \%)\end{array}$ \\
\hline 85 & $\begin{array}{l}2.95 \mathrm{E}-10 \\
(0.92)\end{array}$ & $\begin{array}{c}3.38 \mathrm{E}-10 \\
(1.05)\end{array}$ & $\begin{array}{c}3.13 \mathrm{E}-10 \\
(0.97)\end{array}$ & $\begin{array}{c}3.22 \mathrm{E}-10 \\
(7.88 \%)\end{array}$ \\
\hline 90 & $\begin{array}{c}3.70 \mathrm{E}-11 \\
(0.87)\end{array}$ & $\begin{array}{c}4.13 \mathrm{E}-11 \\
(0.97)\end{array}$ & $\begin{array}{c}3.74 \mathrm{E}-11 \\
(0.88)\end{array}$ & $\begin{array}{c}4.24 \mathrm{E}-11 \\
(9.53 \%)\end{array}$ \\
\hline
\end{tabular}

*MCNP6 calculation is assumed to be reference data.

hand, the new data sets stabilize as penetration depth increases, despite a slight overestimation at a shallow penetration depth. Also, the buildup factors derived from MCNP6.1 code produce more accurate calculation results than those from the other Monte Carlo code. From these results, it was confirmed that gamma-ray buildup factors are sufficiently evaluated from the Monte Carlo codes with the latest crosssection library, which produces reasonable results at a deep penetration depth.

\section{Conclusions}

The gamma-ray buildup factors of a single iron material are derived using the most widely used Monte Carlo Codes (MCNP6.1 and MCNP5-1.60) and ENDF/B-VI.8. Simple benchmark calculation confirms that existing data cause an underestimation in the results as the penetration depth increases, while new data draw stable results overall, except for a slight overestimation at a shallow penetration depth. Therefore, it is likely that gamma-ray buildup factors for existing and new compound materials can be sufficiently re-evaluated from the Monte Carlo codes with the latest cross-section library.

\section{References}

[1] K.A. Litwin, I.C. Gauld, and G.R. Penner, Improvements to the Point Kernel Code QAD-CGGP: A Code Validation and User's Manual, COG-94-65, AECL, 1994

[2] D.K. Trubey, New Gamma-Ray Buildup Factor Data for Point Kernel Calculations: ANS-6.4.3 Standard Reference Data, NUREG/CR-5740, 1991

[3] D.B. Pelowitz (Ed.), MCNP6 User's Manual Version 1.0, LA-CP-13-00634, LANL, 2013

[4] X-5 Monte Carlo Team, MCNP-A General Monte Carlo N-Particle Transport Code, Version 5, LA-CP-03-0245, LANL, 2003

[5] J. K. Shultis and R. E. Faw, Radiation Shielding, ANS, 2000

[6] Morgan C. White, Further Notes on MCPLIB03/04 and New MCPLIB63/84 Compton Broadening Data For All Versions of MCNP5, LA-UR-12-00018, LANL, 2012 